anmeldungen der "Farbenfabriken vorm. Friedr. Bayer \& Co.\& sind die Benzidinabkömmlinge mit substituirenden Gruppen in der Orthostellung zu den Amidogruppen als Metaderivate bezeichnet, also die Verbindung

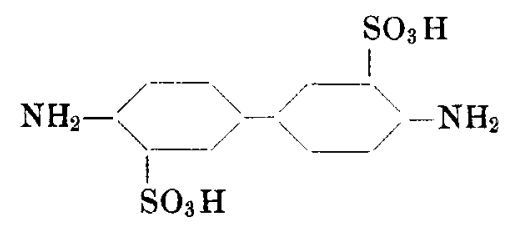

als Benzidin- $m$-disulfosäure.

Mag eine solche Bezeichnungsweise immerhin gerechtfertigt sein durch die Analogie mit den entsprechenden Toluidinderivaten, so kann es doch nur Verwirrung hervorrufen, wenn die herkömmliche Nomenclatur einfach verlassen und, ohne besonders darauf aufmerksam zu machen, eine neue befolgt wird. Zudem hat die ältere Bezeichnung den Vorzug grösserer Durchsichtigkeit. Die Namen $o$ - und $m$-Diamidobenzidin deuten schon die Aehnlichkeit der bez. Verbindungen mit $o$ - resp. $m$-Phenylendiamin an und anch aus diesem Grunde empfiehlt es sich wohl vorläufig an der alten Nomenclatur festzuhalten, die auch in der 2. Auflage von Beilstein's Handbuch beibehalten ist.

Organ. Laboratorium der techu. Hochschule zu Berlin.

542. G. Kraemer, A. Spilker und P. Eberhardt: Ueber die Styrolverbindungen der Benzolkohlenwasserstoffe und ihr Uebergang in Anthracen und methylirte Anthracene.

(Eingegangen am 15. November.)

\title{
2. Theil.
}

Für die in dem vorigen Heft dieser Berichte schon cursorisch erwähnten Versuche diente das aus synthetisch gewonnener Zimmtsäure hergestellte Styrol, da die Abscheidung des Styrols aus dem Robxylol selbst eine zu umständliche und zeitraubende war.

Zum Erhalt des Styrols verfuhren wir wie folgt:

Je $1 \mathrm{~kg}$ rohe Zimmtsäure wurde mit schwacher Flamme und so langsam, dass etwa 4-5 Stunden zu jeder Operation nöthig waren, aus einer $4 \mathrm{~L}$ fassenden Kupferblase unter Anwendung eines gut wirkenden Kühlers abdestillirt. Man erhielt ca. $360 \mathrm{gr}$ Rohstyrol, das 
zur Befreiung von Metastyrol zunächst einmal in luftverdünntem Raume dann bei normalem Drueke fractionirt wurde und dabei $320 \mathrm{gr}$ oder 45.5 pCt. der theoretischen Ausbeute an reinem Styrol gab.

Ein Gemisch aus dem vorwiegend aus Metaxylol bestehenden Reinxylol des Handels mit $5 \mathrm{pCt}$. dieses Styrols gab genau dasselbe Product, das aus dem Rohxylol géwonnen war.

Wir unterwarfen weiter die drei isomeren Xylole in reinem $\mathrm{Zu}$ stande, dann das Pseudocumol, das Toluol und endlich auch mit negativem Erfolge das Benzol derselben Reaction.

Nach der im ersten Theile dieser Abhandlung ${ }^{1}$ ) für die Condensation aufgestellten Formel wärden die entstehenden Körper $\alpha, \gamma$-Substitutionsproducte des Propans sein. Um aus ihnen die bereits mitgetheilte Umwandlung in Anthracenderivate zu erklären, müsste man annehmen, dass aus der Mitte des Molecüls, aus der Verbindungskette der zwei Benzolringe, ein C-Atom herausbräche, so dass das Molecül beim Ueberhitzen in zwei Theile zerfallen und von neuem zusammentreten müsste. Die ausserordentlich guten Ausbeuten bei der Bildung des Methylanthracens wären aber bei einer so complicirten Reaction mindestens auffallend.

In der Sitzung vom 10. November machten nun die Herren Professoren Pinner und Liebermann darauf aufmerksam, dass die Condensation ebenso gut nach folgendem Schema verlaufen könne:

$$
\begin{aligned}
\mathrm{C}_{6} \mathrm{H}_{5} \cdot \underset{\mathrm{C}}{\mathrm{CH}}+\frac{\mathrm{SO}_{4} \mathrm{H}+\mathrm{H}}{\mathrm{H}} & \mathrm{CH}_{2} \cdot \mathrm{C}_{6} \mathrm{H}_{4}\left(\mathrm{CH}_{3}\right) \\
= & \mathrm{C}_{6} \mathrm{H}_{5} \cdot \underset{\mathrm{CH}}{\mathrm{CH}} \cdot \mathrm{CH}_{3} \cdot \mathrm{C}_{6} \mathrm{H}_{4}\left(\mathrm{CH}_{3}\right)
\end{aligned}
$$

Dass also statt $\alpha, \gamma$-, $\alpha$-, $\beta$-Propanderivate entständen. Es würde sich hieraus nach folgendem Schema die Bildung von Anthracenderivaten sehr leicht erklären:

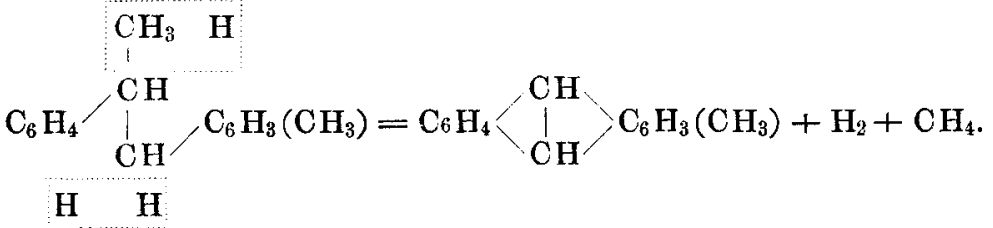

In der That bietet dieses Bild so viel Wahrscheinliches und Verlockendes, dass wir nicht anstehen, die im Folgenden beschriebenen Körper als $\alpha, \beta$-Propanderivate aufzufassen.

1) Diese Berichte XV, 3170 . 


$$
\begin{aligned}
& m \text {-Xylolstyrol }=\alpha \beta \text {-Phenyl-m-Toluylpropan, } \\
& \mathrm{C}_{6} \mathrm{H}_{5} \cdot \mathrm{CH} \cdot \stackrel{1}{\mathrm{C}} \mathrm{H}_{2} \cdot \mathrm{C}_{6} \mathrm{H}_{4} \cdot \stackrel{3}{\mathrm{CH}_{3}} \\
& \mathrm{CH}_{3} \text {. }
\end{aligned}
$$

Zur Darstellung dieser Verbindung wird in ein Gemisch von $500 \mathrm{~g}$ $m$-Xylol mit $30 \mathrm{~g}$ Styrol nach und nach unter heftigem Schütteln $30 \mathrm{gr}$ concentrirte Schwefelsäure eingetragen. Um gute Ausbeuten zu erzielen darf man die Temperatur des Reactionsgemisches nicht über laue Warme steigen lassen. Nach beendeter Reaction setzt sich die Schwefelsäure, gemischt mit der entstandenen Xylolsulfosäure, als schwere braune Schicht gut ab und kann leicht entfernt werden.

Die überstehende Oelschicht wird mit etwas Wasser, dann mit verdünnter Natronlauge und wieder mit Wasser bis zur neutralen Reaction gewaschen. Durch Abblasen mit Wasserdampf entfernt man das unveränderte Xylol und erhält so als Rückstand gegen $48 \mathrm{gr}$ eines bräunlichen Oeles, das zunächst einmal zur Abscheidung geringer Mengen Metastyrols in luftverdünnten Raume, dann bei gewöhnlichem Drack fractionirt wird. Die Hauptmenge $38 \mathrm{gr}$ geht bei $110 \mathrm{~mm}$ Druck gegen $240^{\circ}$ bei normalem Druck unveräudert bei $298-299^{\circ}$ uncorr. $=311-312^{\circ}$ corr. über und gab bei der Analyse folgende Zahlen:

$\begin{array}{lcc} & \text { Gefunden } & \text { Ber. für } \mathrm{C}_{16} \mathrm{H}_{18} \\ \text { Koblenstoff } & 91.53 & 91.43 \mathrm{pCt} . \\ \text { Wasserstoff } & 8.72 & 8.57 》\end{array}$

Der Körper stellt im reinen Zustande ein wasserhelles Oel dar, das schwach fluorescirt und in der Kälte nicht zum Erstarren zu bringen ist. Mit absolutem Alkohol, Aether, Benzol und dergl. auch mit $\mathrm{Pe}$ trolbenzin ist er in jedem Verhältniss mischbar, in Wasser ist er unlöslich. Sein spec. Gewicht bei $15^{\circ}$ beträgt 0.987 .

Durch Oxydation mit verdünnter Salpetersäure, sowie mit Chromsïure konnten bis jetzt nur harzige Producte erhalten werden. Ebensowenig waren Nitroproducte und Bromderivate in krystallinischem Zustande abzuscheiden. Concentrirte Salpetersäure wirkt ziemlich heftig ein, Brom dagegen nur langsam unter Bromwasserstoffabspaltung.

Versuche um die Vereinigung von Styrol mit Xylol durch andere Condensationsmittel wie Salzsäure, oder durch Erhitzen des Gemisches im Einschlussrohr zu bewirken, führten sämmtlich zu negativen Resultaten. Entweder war das Gemisch unverändert, oder es war das Styrol in Metastyrol übergegangen.

Auch zeigte sich, dass aus einem Gemisch äquimolecularer Mengen der Bestandtheile durch Schwefelsäure nur geringe Quantitäten der Verbinưung entstehen. Die Flüssigkeit erhitzt sich vielmehr sehr stark 
scbon auf Zusatz der ersten Tropfen Schwefelsäure unter Bildung von Metastyrol. Ein Verdünnen mit indifferenten Lösungsmitteln wie Aether und Chloroform gab ebenfalls nur schlechte Ausbeuten.

$$
\text { Methylanthracen. }
$$

Wie das aus Rohxylol enthaltene Phenyltoluylpropan, so gebt auch das aus dem $m$-Xylol entstehende Condensationsproduct beim Leiten durch ein dunkelroth glühendes Rohr fast glatt in Methylauthracen über, das sich in der Vorlage als schwach öliger Krystallkuchen und als Sublimat rorfindet. Durch Destillation unter Anwendung von Wasserdampf oder durch Sublimation und Umkrystallisiren aus Pyridin oder Eisessig wird es in schönen, gelblich-weissen Krystallblättchen erbalten, die bei $202^{\circ}$ uncorr. (207 ${ }^{\circ}$ corr.) schmelzen.

$$
\begin{gathered}
p \text {-Xylol-Styrol }=\alpha, \beta \text {-Phenyl-p-Toluylpropan. } \\
\qquad \mathrm{C}_{6} \mathrm{H}_{5} \cdot \mathrm{CH} \cdot \mathrm{CH}_{2} \cdot \mathrm{C}_{6} \mathrm{H}_{4} \cdot \mathrm{CH}_{3}^{4} \\
\mathrm{CH}_{3}
\end{gathered}
$$

Genau in derselben Weise wie aus dem $m$-Xylol und Styrol wurde auch aus dem $p$-Xylol ein Additionsproduct dargestellt, das in allen seinen Eigenschaften dem ersteren zum Verwechseln äbılich ist. Es siedet bei normalem Druck bei $290-291^{\circ}$ uncorr. (302 bis $3\left(13^{\circ} \mathrm{corr}\right.$.)

Die Analyse ergab:

$$
\begin{aligned}
& \text { Gefunden } \quad \text { Ber. für } \mathrm{C}_{36} \mathrm{H}_{18} \text { : } \\
& \text { Koblenstoff } 91.35 \quad 91.43 \mathrm{pCt} \text {. } \\
& \begin{array}{lll}
\text { Wasserstoff } 8.61 & 8.57 \text { 》 }
\end{array} \\
& \text { o-Xylol-Styrol }=a, \beta \text {-Phenyl-o-Toluylpropan, } \\
& \mathrm{C}_{6} \mathrm{H}_{5} \cdot \mathrm{CH} \cdot \mathrm{CH}_{2}^{1} \cdot \mathrm{C}_{6} \mathrm{H}_{4} \cdot \mathrm{C}^{2} \mathrm{H}_{3} \text {. } \\
& \mathrm{CH}_{3}
\end{aligned}
$$

Zu der bei $m$-Xylol-Styrol angegebenen Darstellungsweise ist eine verbältnissmässig übergrosse Quantität Xylol erforderlich, die freilich zum grössten Theile unverändert wiedergewonnen wird. Da uns aber nur eine kleine Menge, etwa $50 \mathrm{~g}$ wirklich reines 0 -Xylol zur Verfügung stand, suchten wir die Darstellungsmethode etwas zu ändern und erhielten auf folgende Weise recht gute Resultate: $45 \mathrm{~g} o$-Xylol wurden mit $2 \mathrm{~g}$ Styrol gemischt und in die Mischung nach und nach $15 \mathrm{~g}$ concentrirte Schwefelsäure eingetragen.

Nach längerem kräftigen Schütteln liessen wir absitzen, gossen in die fobere Oelschicht unter vorsichtigem Umschwenken weitere $2 \mathrm{~g}$ Styrol, sodass diese sich zunächst mischten, und schüttelten 
erst dann kräftig, um die Schwefelsäure zur Wirkung kommen zu lassen. In dieser Weise wurden nach und nach $15 \mathrm{~g}$ Styrol eingetragen.

Während der ganzen Operation wurde die Flasche unter der Wasserleitung gut gekühlt, dabei natürlich sorgfältig verschlossen, sodass kein Tropfen Wasser in die Flüssigkeit gelangen konnte.

Weiter wurde dann wie früber die Säure getrennt und das Oel gewaschen. Statt mit Wasserdampf abzublasen, kann man zweckmässig sogleich im luftverdünnten Raume fractioniren.

Was bei einem Drucke von $100-150 \mathrm{~mm}$ bei $230-250^{\circ}$ übergegangen ist, wird noch einmal bei normalem Druck fractionirt.

Wir erbielten so aus $15 \mathrm{~g}$ Styrol $28 \mathrm{~g}$ rohes und gegen $20 \mathrm{~g}$ reives Product.

Das $\alpha, \beta$-Phenyl-o-Toluylpropan siedet bei $302-303^{\circ}$ uncorr. $\left(316-317^{\circ}\right.$ corr. $)$ und ist in seinen übrigen Eigenschaften der Metaund Paraverbindung gleich.

$$
\begin{gathered}
\text { Pseudocumol-Styrol= } \alpha, \beta \text {-Phenyl-Xylylpropan, } \\
\mathrm{C}_{6} \mathrm{H}_{5} \cdot \underset{\mathrm{CH}}{\mathrm{CH}} \mathrm{CH}_{3} \cdot \mathrm{C}_{6} \mathrm{H}_{3}\left(\mathrm{CH}_{3}\right)_{2}
\end{gathered}
$$

Das unsymmetrische Trimethylbenzol giebt, mit Styrol in der gleichen Weise wie das $m$-Xylol behandelt, ein Condensationsproduct, das noch leichter und besser zu entstehen scheint. Aus $50 \mathrm{~g}$ Styrol erhielten wir $90 \mathrm{~g}$ rohes und gegen $70 \mathrm{~g}$ reines Phenyl-Xylylpropan, das bei $310^{\circ}$ uncorr. ( $324^{\circ}$ corr.) siedete, im übrigen seinen niederen Homologen glich.

Die Analyse ergab:

$$
\begin{array}{lccc} 
& \multicolumn{2}{c}{\text { Gefunden }} & \text { Ber. für } \mathrm{C}_{17} \mathrm{H}_{20} \\
\text { Kohlenstoff } & 91.11 & 91.14 & 91.07 \mathrm{pCt} . \\
\text { Wasserstoff } & 8.98 & 8.93 & 8.93
\end{array}
$$

Dimethylanthracen.

Durch Ueberhitzen ging das Phenyl-Xylylpropan wie das Toluylderivat in glatter Umsetzung in ein Anthracenderivat, das Dimethylanthracen über. Aus $20 \mathrm{~g}$ desselben wurden $15 \mathrm{~g}$ Dimethylantbracen gewonnen, das nach dem Sublimiren und Umkrystallisiren aus Eisessig bei $298^{\circ}$ uncorr. ( $=235^{\circ}$ corr.) schmolz.

Die Analyse ergab:

\begin{tabular}{cccc} 
& \multicolumn{2}{c}{ Gefunden } & Ber. für $\mathrm{C}_{16} \mathrm{H}_{4}$ \\
Kohlenstoff & 93.12 & 93.07 & $93.21 \mathrm{pCt}$. \\
Wasserstoff & 7.16 & 6.94 & 6.79 D
\end{tabular}




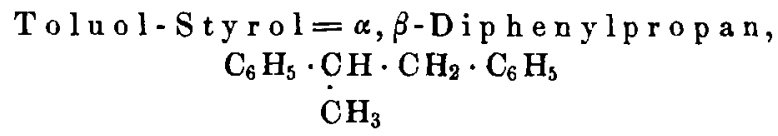

Die Vereinigung von Toluol mit Styrol scheint etwas weniger leicht zu erfolgen wie die der Di- und Trimethylbenzole, wenigstens war die Ausbeute an Diphenylpropan weniger gut. Man muss daher hier die Schwefelsäure tropfenweise zufügen und jede Temperaturerhöhung sorgfältig vermeiden. Aus $40 \mathrm{~g}$ Styrol in $500 \mathrm{~g}$ Toluol gelöst, wurden $55 \mathrm{~g}$ Rohproduct und daraus $35 \mathrm{~g}$ analysenreines Material erhalten. Das nicht in Reaction getretene Styrol fand sich als Metastyrol wieder.

Das Diphenylpropan, ein wasserhelles öliges Liquidum, siedet Lei $280-282^{\circ}$ uncorr. (291-2930 corr.).

Die Analyse ergab:

\begin{tabular}{lccc} 
& \multicolumn{2}{c}{ Gefunden } & Ber. für $\mathrm{C}_{15} \mathrm{H}_{16}$ \\
Kohlenstoff & 91.83 & $91.89 \mathrm{pCt}$. & $91.83 \mathrm{pCt}$ \\
Wasserstoff & 8.47 & 8.41 & 8.17
\end{tabular}

Anthracen.

Während die Bildung des Mono- and besonders des Dimethylanthracens aus den entsprechenden Propanderivaten in glatter Reaction verlief, erbielten wir als Ueberhitzungsproduct des Diphenylpropans ein schmieriges Oel, aus dem kaum ein krystallinisches Product abzuscheiden war. Durch Oxydation des Rohproductes mit Chromsäure wurde allerdings ein gut krystallisirtes Anthrachinon erhalten, das durch seinen Schmelzpunkt und seine Reactionen identificirt wurde, jedoch betrug die daraus berechnete Antbracenmenge nur wenige Procente der theoretischen Ausbeute.

Wir vermutheten den Grund dieser Erscheinung darin, dass das Anthracen bei seiner Entstehungstemperatur sogleich weiter zerfalle. Ein Versuch, bei dem reines Anthracen in Benzollösung überhitzt wurde, bestätigte diese Annahme. Von dem angewendeten Anthracen konnten nur etwa $9 \mathrm{pCt}$. in dem Reactionsproduct wieder aufgefunden werden. Ein gleicher Versuch mit Methylanthracen gab dagegen fast die gesammie Menge desselben unverăndert zurück. Somit ist die geringe Ausbeate an Anthracen und die so vorzügliche bei der Synthese der methylirten Anthracene erklärt.

\section{Benzol und Styrol.}

Benzol mit Styrol in derselben Weise zu combiniren, wie das mit seinen Homologen geschehen war, gelang nicht. Mehrere Versuche, die mit peinlichster Sorgfalt ausgeführt wurden, ergaben das Benzol unverändert, das Styrol völlig in Metastyrol übergefübrt. Auch Abänderungen der Versuchsbedingungen führten nicht zum Ziel. Es ist 
danach eine Seitenkette, (Methylgruppe) für die Reaction erforderlich, sie vermittelt die Bindung, und die entstehenden Körper sind denınach Derivate des Propans.

Die Eigenschaften des von uns aus Tolnol und Styrol erhaltenen Körpers lassen sich sowohl mit den in der Litteratur über das $\alpha, \beta$, als auch mit den über das $\alpha, \gamma$-Diphenylpropan vorhandenen Angaben in Einklang bringen. Diese Angaben erstrecken sich vor allem auf den Siedepunkt, der für das ans Propylenchlorid und Benzol dargestellte $\left(x, \beta\right.$-Derivat von Silra $\left.{ }^{1}\right)$ zu $277-279^{\circ}$ und für das $\alpha, \gamma$ Diphenylpropan von $\mathrm{Graebe}^{2}$ ) and von Cla us und Mercklin ${ }^{3}$ ) zu $290-300^{\circ}$ angegeben wird. Die letzteren stellten diesen Körper aus Trichlorbydrin und Benzol bei Gegenwart von Aluminiumchlorid, Graebe denselben aus Dibenzylketon durch Reduction dar. Wir selbst fanden für unsere Verbindung den Siedepunkt $280-282^{\circ}$ uncorr. $=291-293^{\circ}$ corr. Bei dieser hohen Temparatur können derartig geringe Differenzen nicht als Entscheid für oder gegen die Identität $\mathbf{z w e i e r}$ Körper dienen; dieser würde vielmehr erst durch die Untersuchung der Ueberhitzungsproducte eines $\alpha, \beta$ - und eines $\alpha, \gamma$-Phenyltoluylpropaus erbracht werden, deren Constitution selbst aus ihrer Synthese unzweifelhaft hervorgebt.

Die von J. Weil er und O. Fischer ${ }^{4}$ ) dargestellten Körper der Formel $\mathrm{C}_{15} \mathrm{H}_{16}$ und $\mathrm{C}_{16} \mathrm{H}_{18}$, die beim Ueberhitzen ebenfalls Metbylanthracen gaben, sind mit unseren Verbindungen nur isomer, nicht identisch. Es gelang deshalb auch nicht, aus diesen die Brom- und Nitroverbindungen, sowie Oxydationsproducte zu gewinsen, die aus jenen erhalten waren. Wie schon oben erwähnt, führten bei dem Phenyltoluylpropan Oxydationsversuche mit Chromsäure und mit verdünnter Salpetersäure zu keinem fassbaren Resultat. Aus dem Diphenylpropan wurde durch Kaliumpermanganat in wässeriger Lösung, das erst beim Erhitzen einwirkte, eine Säure gewonnen, die gegen $140^{\circ}$ schmolz, bis jetzt aber nicht eingehender untersucht wurde.

Ob auch Benzolhomologe mit längeren Seitenketten, Aethylbenzol, Propylbenzol u. s. w. sowie andere Kohlenwasserstoffe mit Seitenketten, wie Methylanthracen, Methylnaphtalin a. dgl. der Verbindung mit Styrol unter der Einwirkung von Schwefelsäure fähig sind, bleibt noch za untersuchen, ist jedoch höchst wabrscheinlich. Ein flüchtiger Versuch, Styrol mit $m$-Kresol zu vereinigen, scheint in der That zu einem Condensationsproducte geführt zu haben. Der entstandene Körper wird voraussicbtlich beim Ueberhitzen Oxyanthracen geben.

1) Compt. rend. 89, 606 .

2) Diese Berichte VII, 1627.

3) Diese Berichte XVIII, 2935.

4) Disse Berichte VII, 1181 u. f. 
Auf die Verfolgung der Untersuchung der hierbei entstehenden Körper haben wir Verzicht geleistet, als uns bekannt wurde, dass Dr. Königs in dieser Richtung schon Erfolge erzielt hat, und weitere Aufschlüsse darüber baldigst in Aussicht stehen. Die von diesem Forscber schon verwirklichte Bindung des Phenols mit Styrol beweist, dass zur Herbeiführung derselben recht wohl auch der Wasserstoff des Benzolringes dienen kann. Das Studium der Styrolkresolverbindungen wird also auf alle Fälle zu recht interessanten $\Lambda$ ufschlüssen führen über die Frage der Reactionsfühigkeit von Wasserstoffatomen in verschiedener Stellung.

Erkner, im November 1890.

\section{G. Krâmer und A. Spilker: Uober das Inden und Styrol im Steinkohlentheer.}

(Vorgetragen von G. Krämer.)

Am Schlusse unserer Abhandlung ${ }^{1}$ ) über Cumaron ist schon erwähnt worden, dass in den höher siedenden Antheilen der leichten Oele des Steinkoblentheers dem Cumaron verwandte Körper rorkommen, deren Reindarstellung damals noch gewissen Schwierigkeiten begegnete, die sich inzwischen haben beseitigen lassen. Wir schicken zunächst voraus, dass wir den umständlichen Weg über die Bibromverbindung dieser Körper, wie er anfangs zum Erhalt des Cumarons beschritten werden musste, sehr bald haben verlassen können, da sich herausstellte, dass mittelst der Pikrinsäureverbindungen, die Trennung von den übrigen Begleitern einfacher verwirklicht werden konnte.

Ueber die Darstellung des Cumarons nach diesem Verfahren finden sich in dem ertheilten D. R.-P. No. 53792 die nöthigen Angaben und tragen wir hier nur nach, dass der Schmelzpunkt der Pikrinsäureverbindung bei $102-103^{\circ}$ gefunden wurde.

Im Wesentlichen konnte auch für die Gewinnung des neuen Körpers dasselbe Verfahren beibehalten werden, nur ist die Herstellung des Rohmaterials etwas schwieriger, weil einerseits die cumarouhaltigen Fraktionen, andererseits das Naphtalin durch sorgfältiges Fraktioniren möglichst gan $z$ beseitigt sein müssen. Man löst in der zu $90 \mathrm{pCt}$. zwischen $176-182^{\circ}$ (uncorr.) übergehenden Fraktion des

1) Diese Berichte XXIII, 78. 